\title{
International initiative takes AIM at resistant bacteria
}

- Mark Greener -

The rapid spread of severe acute respiratory syndrome (SARS) worldwide, borne by international travellers, is a timely reminder that infections do not respect borders. Similarly, resistant bacteria pose a growing problem, clinically and economically, across the world. And international problems need international answers. So, a new initiative is taking aim at resistant bacterial infections by encouraging a radical change in prescribing practices worldwide. The Academy for Infection Management (AIM), supported by an educational grant from AstraZeneca, is a global educational programme that brings together leading experts from a variety of specialities. The academy has two aims. First, AIM will "address the current crisis in hospital morbidity and mortality that has arisen partly as a result of inappropriate use of antibiotics". Second, AIM plans to "radically change the treatment paradigm for serious nosocomial infections to improve patient outcomes and save lives".

"Growing antimicrobial resistance and inappropriate antibiotic use has lead to a crisis in hospital infection control. Nosocomial infections are a serious problem and have severely impeded our ability to fight disease resulting in thousands of unnecessary deaths. The problem is going to get worse unless we radically change our prescribing practices - doctors need to recognize that the antibiotics they select can impact whether a patient lives or dies", said Dr Marin Kollef, chair of AIM and associate professor at the Department of Internal Medicine, Washington University School of Medicine, St Louis, Missouri. "The Academy for Infection Management's mission is to address this crisis by educating clinicians about radically changing the treatment paradigm for nosocomial infections, which involves the early and empiric treatment of infections. A wealth of evidence has proved that this can significantly improve infection control and save lives", Dr Marin said.

\section{A worldwide problem}

"Resistance is a common, world wide problem", added Dr Robert Masterton, medical director at Ayrshire and Arran Acute Hospital Trust, UK, at the launch of AIM in Barcelona, Spain, on 26 February 2003. "It's depressing that we don't seem to affect this rate. The problem is here to stay", he said. He recited a liturgy of resistant bacteria that pose significant clinical problems, including methicillin-resistant Staphylococcus aureus (MRSA), vancomycin-resistant Enterococcus (VRE), vancomycin-intermediate Staphylococcus aureus (VISA) and multi-drug resistant (MDR) tuberculosis.

The latter poses a problem even in the developed world. For example, a recent paper examined 25217 isolates of Mycobacterium tuberculosis complex collected between 1993 and 1999 in the UK. Of these, $6.1 \%$ were resistant to at least one drug, while $5.6 \%$ were resistant to isoniazid either alone or with other antibacterial drugs. Worryingly, $1.2 \%$ of $M$. tuberculosis isolates were resistant to several drugs. Furthermore, the numbers of drug-resistant $M$. tuberculosis isolates increased between 1993 and 1999. Young men, people living in London, patients born overseas, those with a history of tuberculosis and HIV-positive subjects were the most likely to be infected with drug-resistant tuberculosis.

Clinically, Dr Masterton noted that antibacterial resistance increases the chance that physicians will prescribe inappropriate therapy, which is an independent risk factor for increased mortality. For example, bacteraemia and septicaemia are associated with a mortality rate, even in hospital, of $30-40 \%$. In one study, the hospital mortality rate of critically ill patients with bloodstream infections receiving inappropriate antibacterial drugs was $61.9 \%$. This compared to $28.4 \%$ among those managed with adequate therapy.

Antibacterial resistance is also associated with increased morbidity, mortality and duration of hospitalisation. As a result, patients require more toxic and more expensive therapy. "The costs are high and they are rising", Dr Masterton commented. For example, an increasing proportion of hospital-acquired infections are now resistant to at least one antibacterial. Dr Masterton commented that hospital-acquired infections are responsible for 5000 deaths annually in the UK and cost the NHS around $£ 1$ billion. Tragically, some $30 \%$ of hospital-acquired infections could be prevented, estimated Dr Masterton.

\section{A staggering cost}

Against this background, Dr Peggy McKinnon, director of Infectious Disease Outcomes, Wayne Street University, Detroit, US, added that "the cost of antibiotic resistance is staggering". Dr McKinnon noted that antibacterials account for $10-40 \%$ of the drug budget in hospitals. However, she noted that up to $40 \%$ of antibacterials are misused, which helps drive the growth of resistance. In turn, the treatment of resistant organisms increases costs and the length of hospital stay.

Reducing the costs associated with resistance may mean "using expensive drugs first", Dr McKinnon said. She suggested that "pharmacoeconomic analyses of infection treatment must consider all cost inputs and outcomes resulting from treatment". For instance, she noted that "drug acquisition cost is a minimal component of the total cost". In contrast, Dr McKinnon argued that hospitalisation represented the single greatest component of the total cost of resistant infection. Indeed, she told delegates at the launch that, according to the American Hospital Association, one day in hospital attributable to treatment of infection costs between \$US800 and \$US1600. Overall, she suggested that "unnecessary" costs associated with resistance in the US reach \$US4 billion each year. "Employing effective empiric regimens and adequate dosing regimens would improve patient outcomes, reduce development of resistance and avoid costs associated with treatment failures and antibiotic resistance", she said.

\section{Combating the spread of resistance}

Against this background, Dr Masterton outlined several elements in a system to combat the spread of resistance. First, health services need to implement surveillance systems to facilitate early warning and action against resistance. Also, "we need prudent antimicrobial prescribing to preserve our antibiotic armamentarium and improve outcomes as well as enhanced infection control to reduce resistance spread 


\section{International initiative takes AIM at resistant bacteria - continued}

continued

and nosocomial infections", he said. Finally, he advocated using appropriate empirical antibacterials early and then tailoring antibacterial therapy based on culture results.

Bacterial resistance poses a clear and present danger to the antibacterial miracle that helped control oncefeared infections since the Second World War. Currently, pharmacologists are managing - only just, in some cases - to stay one step ahead of the spread of antibacterial resistance. There are a number of possible molecular targets that could lead to a new generation of antibacterials. In the meantime, AIM hopes that its strategy of "early administration of broad-spectrum antimicrobial treatment to high-risk patients" followed by "the rapid tailoring or discontinuation of antimicrobial regimens based on culture results and the clinical course of the patient" should help contain the clinical and economic costs associated with antibacterial resistance.

800888484 